\title{
Fast Figuring of Large Optics by Reactive Atom Plasma
}

\author{
Marco Castelli $^{* a}$, Renaud Jourdain ${ }^{\mathrm{a}}$, Paul Morantz ${ }^{\mathrm{a}}$, Paul Shore ${ }^{\mathrm{a}}$ \\ ${ }^{a}$ Cranfield University, Precision Engineering Institute, Cranfield, MK43 OAL, United Kingdom
}

\begin{abstract}
The next generation of ground-based astronomical observatories will require fabrication and maintenance of extremely large segmented mirrors tens of meters in diameter. At present, the large production of segments required by projects like E-ELT and TMT poses time frames and costs feasibility questions. This is principally due to a bottleneck stage in the optical fabrication chain: the final figuring step. State-of-the-art figure correction techniques, so far, have failed to meet the needs of the astronomical community for mass production of large, ultra-precise optical surfaces. In this context, Reactive Atom Plasma (RAP) is proposed as a candidate figuring process that combines nanometer level accuracy with high material removal rates. RAP is a form of plasma enhanced chemical etching at atmospheric pressure based on Inductively Coupled Plasma technology. The rapid figuring capability of the RAP process has already been proven on medium sized optical surfaces made of silicon based materials. In this paper, the figure correction of a 3 meters radius of curvature, $400 \mathrm{~mm}$ diameter spherical ULE® mirror is presented. This work demonstrates the large scale figuring capability of the Reactive Atom Plasma process. The figuring is carried out by applying an in-house developed procedure that promotes rapid convergence. A $2.3 \mu \mathrm{m} \mathrm{p}-\mathrm{v}$ initial figure error is removed within three iterations, for a total processing time of 2.5 hours. The same surface is then re-polished and the residual error corrected again down to $\lambda / 20 \mathrm{~nm}$ rms. These results highlight the possibility of figuring a metre-class mirror in about ten hours.
\end{abstract}

Keywords: Large optical components, figuring, tool-path algorithms, plasma etching, optical fabrication, extremely large telescopes.

\section{INTRODUCTION}

Since the invention of telescopes four hundred year ago, one of the main driving factors for astronomical discoveries has been the size of their aperture which improves optical resolution and light collection. The history of these instruments has often been illustrated by a trend of diameter increase of primary mirrors over the centuries [1]. This enhancement in observation capability can be easily associated with conceptual breakthroughs in understanding astrophysical phenomena - from the first observation of Jupiter's moons to the measurement of the Hubble constant. In the past two decades, in addition to remarkable images of our universe, the first generation of very large (8-10 m diameter) earth and space based observatories (VLT, Keck, Gemini, Hubble) has been delivering ground-breaking discoveries in the fields of cosmology, exo-planets search, as well as fundamental physics. However, while answering some of the questions "nagging" astrophysicists today, such findings have often generated further scientific queries. As a consequence, in the last few years, so-called giant telescopes with primary mirror diameters ranging from 20 to $100 \mathrm{~m}$ have been proposed. The scientific reasons for these audacious concepts are linked both to the need for even higher resolution and to the fact that no other instrumentation improvement is possible. Citing Gilmozzi (2004) [1], "Now that detectors are at efficiencies close to $100 \%$, large improvements can be obtained only through large increases in diameter." This statement is supported by the progresses made by Adaptive Optics systems that have already enabled near-limit instrumental resolution. Examples of ground based giant telescopes are E-ELT (European Extremely Large Telescope) [2], [3], TMT (Thirty Meters Telescope) [4] and GMT (Giant Magellan Telescope) [5], [6]. Among space observatories, it is worth mentioning the James Webb Space Telescope (JWST), as well as a series of gravity science missions [7], [8].

Clearly, the limiting factors for the fabrication of such huge optical ensembles are the time and cost frames involved for the production of the required large number of ultra-precise specular surfaces. Established manufacturing chains that were successful at producing segmented mirrors for the Kecks [8] are today required to drastically reduce the fabrication times down to 10 hours duration per processing step for a meter-class component. At present, the fine figure correction constitutes the bottleneck stage, as state-of-the-art technologies like Ion Beam Figuring (IBF) [9], Magnetorheological

*m.castelli@cranfield.ac.uk; phone 441234750111 ext. 2796; fax 44 1234 752946; www.cranfield.ac.uk. 
Finishing (MRF) [10] or CNC polishing processes [11] still need tens to hundreds of hours to meet requirements. An alternative solution proposed itself with the development of a dry chemical etching process called Reactive Atom Plasma (RAP). This atmospheric pressure figuring process presents the advantages of a non-contact tool combined with high material removal rate and repeatability at nanometer level. Its rapid figuring capability has been previously demonstrated on surface areas up to $140 \mathrm{~mm}$ diameter [12]. In this paper, successful scaling-up of the RAP process to an area of 400 $\mathrm{mm}$ diameter is demonstrated, thus showing the actual potential for large segment class figuring. An initial figure error of $\sim 2.4 \mu \mathrm{m} \operatorname{PVr}$ [13] (373 $\mathrm{nm} \mathrm{rms})$ was reduced to $280 \mathrm{~nm} \operatorname{PVr}(31 \mathrm{~nm} \mathrm{rms})$ within five iterations. The mean processing time per iteration was $\sim 50$ minutes.

\section{REACTIVE PLASMA FIGURING}

\subsection{Helios 1200}

In Figure 1, a photograph (left) and a configuration sketch (right) of the figuring facility Helios 1200 are shown. Installed in the Loxham lab of Cranfield University Precision Engineering, the machine is conceived for the rapid figuring of optics up to $1.2 \mathrm{~m}$ diameter. Its micrometer resolution motion is ensured by a computer numerical control motion system (Fanuc 30i series). It integrates dedicated software and a double skin structure to increase machine safety. Three axes form the motion configuration. The component, mounted onto a holding carrier (Figure 2, left), moves face-down along the YY direction, while scanned by the plasma torch travelling along free-form paths in the XZ plane (Figure 1, right).
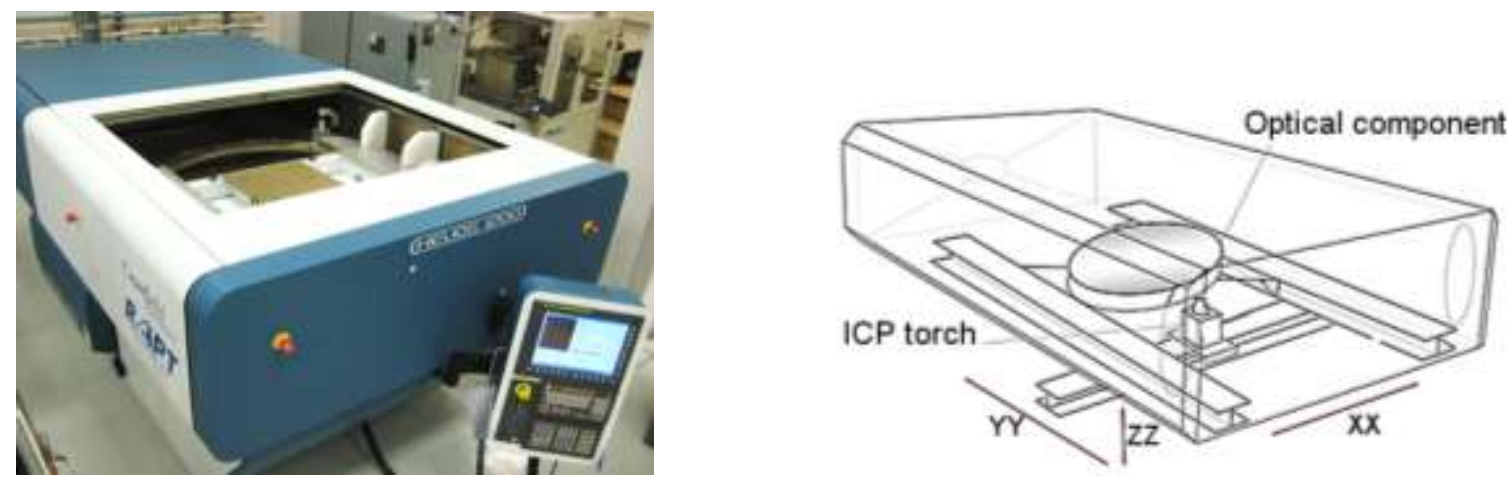

Figure 1. left: Helios 1200 figuring facility in the Loxham Lab of Cranfield University Precision Engineering; right: Sketch of the machine structure.
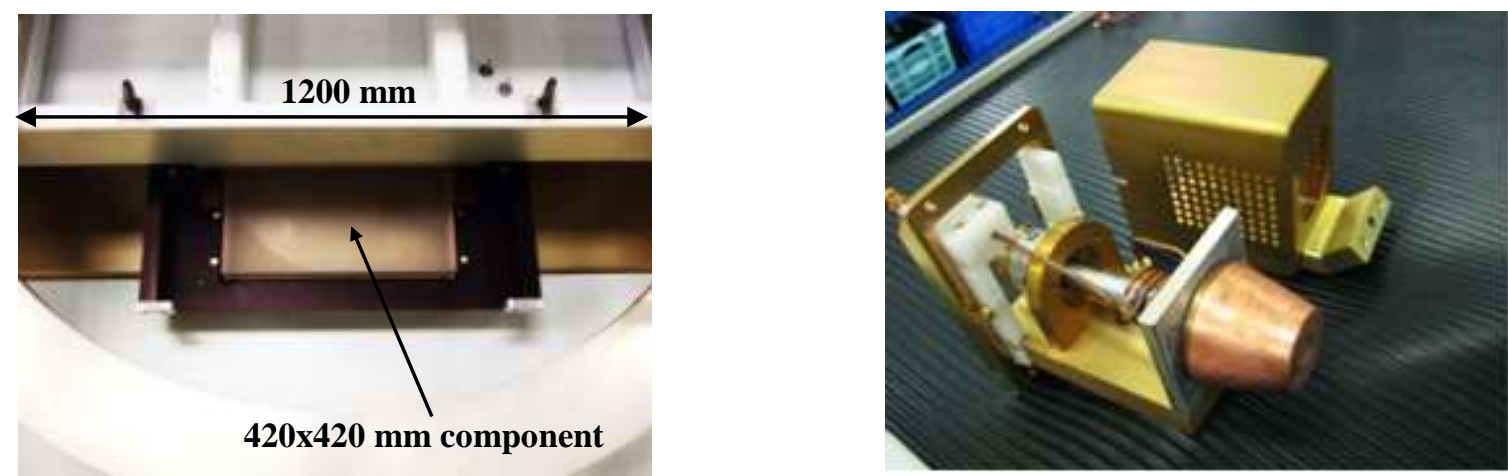

Figure 2. left; Optical component mounted onto the holding carrier; right: : ICP torch in use on Helios 1200.

The material removal is ensured by an inductively coupled plasma torch specially engineered to reduce the heat flux transferred onto the substrate surface using a large water-cool brass nozzle. This torch is based on a De-Laval type design which increases the tool efficiency. (Figure 2, right). The produced etching footprint has a characteristic nearGaussian shape. Depending on the standoff distance between the substrate and aperture of the torch nozzle, Gaussian 
profiles of different width can be imprinted onto a surface, thus varying the figuring authority. In terms of Full Width at Half Maximum (FWHM), the current footprint measures $\sim 11 \mathrm{~mm}$.

\subsection{Tool-path algorithm}

Recent research on Reactive Plasma Figuring has produced high quality figure correction results down to $\lambda / 40 \mathrm{rms}$ on fused silica surfaces [12]. These results were obtained by applying an in-house developed tool-path algorithm, based on a staggered meander-type motion and dwell-time calculations. This raster-path is characterized by a larger main pitch along the YY-direction (Figure 3, right); the same pattern is then alternately reversed and shifted by a smaller secondary pitch to cover all the foreseen passes. The secondary pitch is typically chosen as a divisor of the main one to yield an even number of overturning. For the work presented in this paper, main and secondary pitch sizes were chosen on the basis of previous experimental results.

The design of the described tool-path has the purpose of promoting homogeneity of surface temperature distribution and, consequently, improving etching uniformity. Indeed, surface temperature is a key factor since the removal rates of this chemical etch process are known to increase according to an Arrhenius-type trend. This requires an additional compensation factor to be applied for each iteration steps (see also [12]).
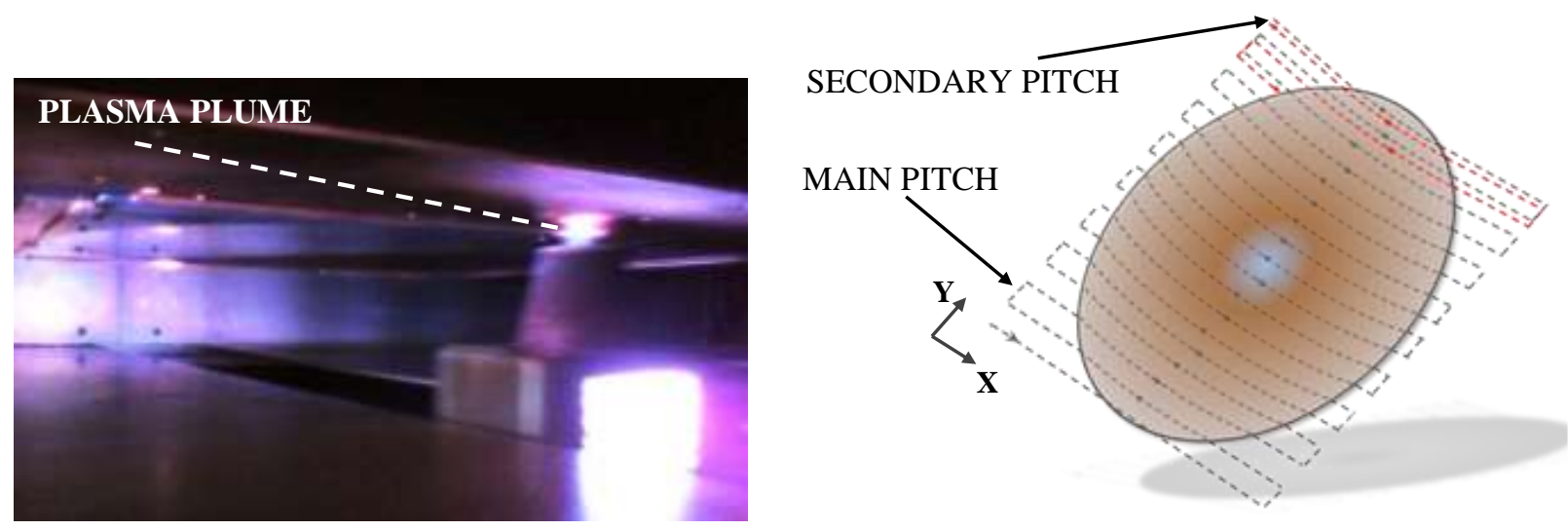

Figure 3. left: RAP torch scanning the 420mmx420mm ULE mirror; right: Schematic of the reversed-staggered tool-path applied to the spherical mirror surface.

In Figure 3 (left), a snapshot of the torch raster-scanning the surface and following the part geometry is shown. The nearby sketch illustrates the application of the tool-path to the correspondent concave spherical surface. The torch follows the part geometry at tangential speeds dictated by a velocity map. This is derived from the measured figure error by means of modified dwell-time methods that account for the non-linear nature of the etching rate [12]. In this work, the iterative figuring procedure, in combination with the described tool-path algorithm, was proven to deliver rapid convergence of the process also on large scale surfaces. This work is detailed and discussed in the next section.

\section{FIGURE CORRECTION OF LARGE OPTICAL SURFACE}

\subsection{Surface preparation}

The blank utilized in this work was a 420 mm x420mm x40mm ULE substrate. In the center of one $420 \mathrm{~mm} \times 420 \mathrm{~mm}$ flat area, a circular region of $400 \mathrm{~mm}$ diameter was ground to a concave spherical shape of $3 \mathrm{~m}$ radius of curvature. The process was performed at Cranfield University Precision Engineering on the BoX grinding machine (Figure 4, left), designed for rapid and accurate grinding of large telescope mirror segments [14]. The form accuracy after grinding, measured on a coordinate measurement machine, was $2.2 \mu \mathrm{m} \mathrm{p}-\mathrm{v}$ (Figure 4, right). Subsequently, the surface was handpolished to improve the roughness and to obtain specular surface suitable for interferometric measurement purposes. In particular, such polishing wasn't intended for subsurface damage removal which is known to influence the post-plasmaetching surface texture [15].

The figure error map after polishing was acquired by means of a vibration insensitive interferometer mounted on top an optical test-tower, designed and assembled in Cranfield (Figure 5, left). Dedicated to for large optics metrology, the repeatability of this setup was proven to be $\sim 30 \mathrm{~nm}$ p-v. The initial surface figure error can be observed in Figure 6 (left). 
The topography map after tip-tilt and power subtraction consisted of a near-astigmatic term of $\sim 2.4 \mu \mathrm{m}$ PVr $(373 \mathrm{~nm}$ rms). This was utilized for the tool-path generation of the first figure correction iteration. This is described in the next section.
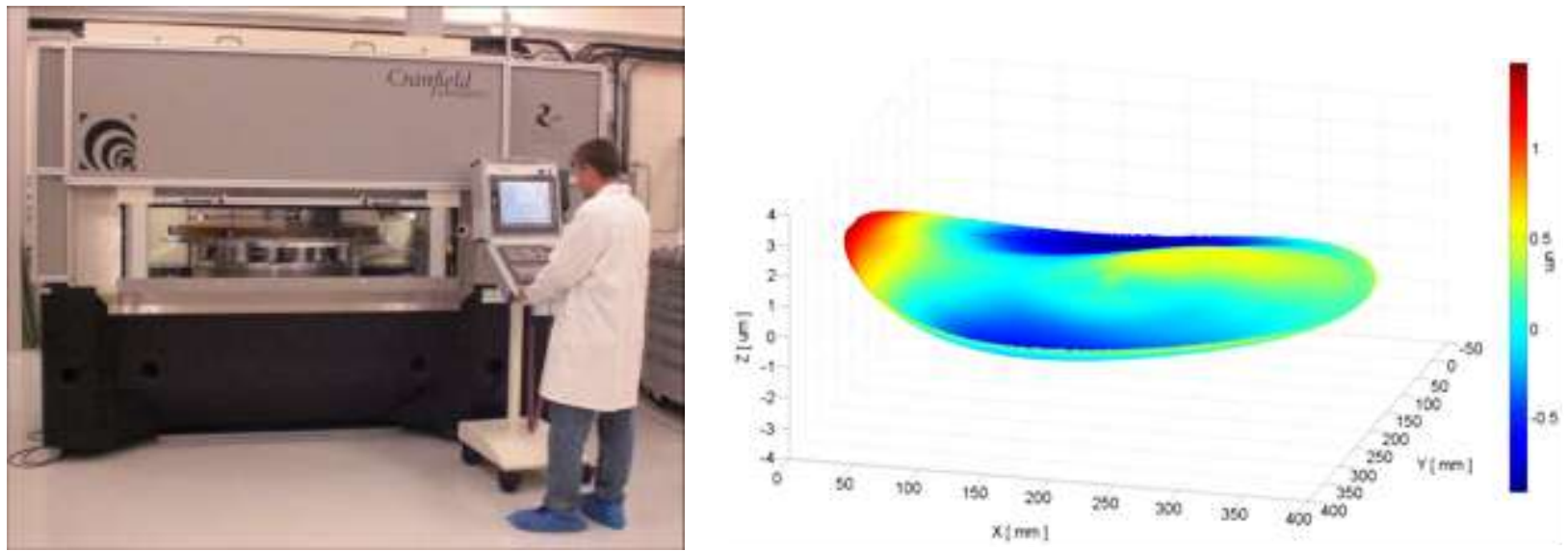

Figure 4. left: BoX grinding machine; right: ULE mirror surface form accuracy after grinding.
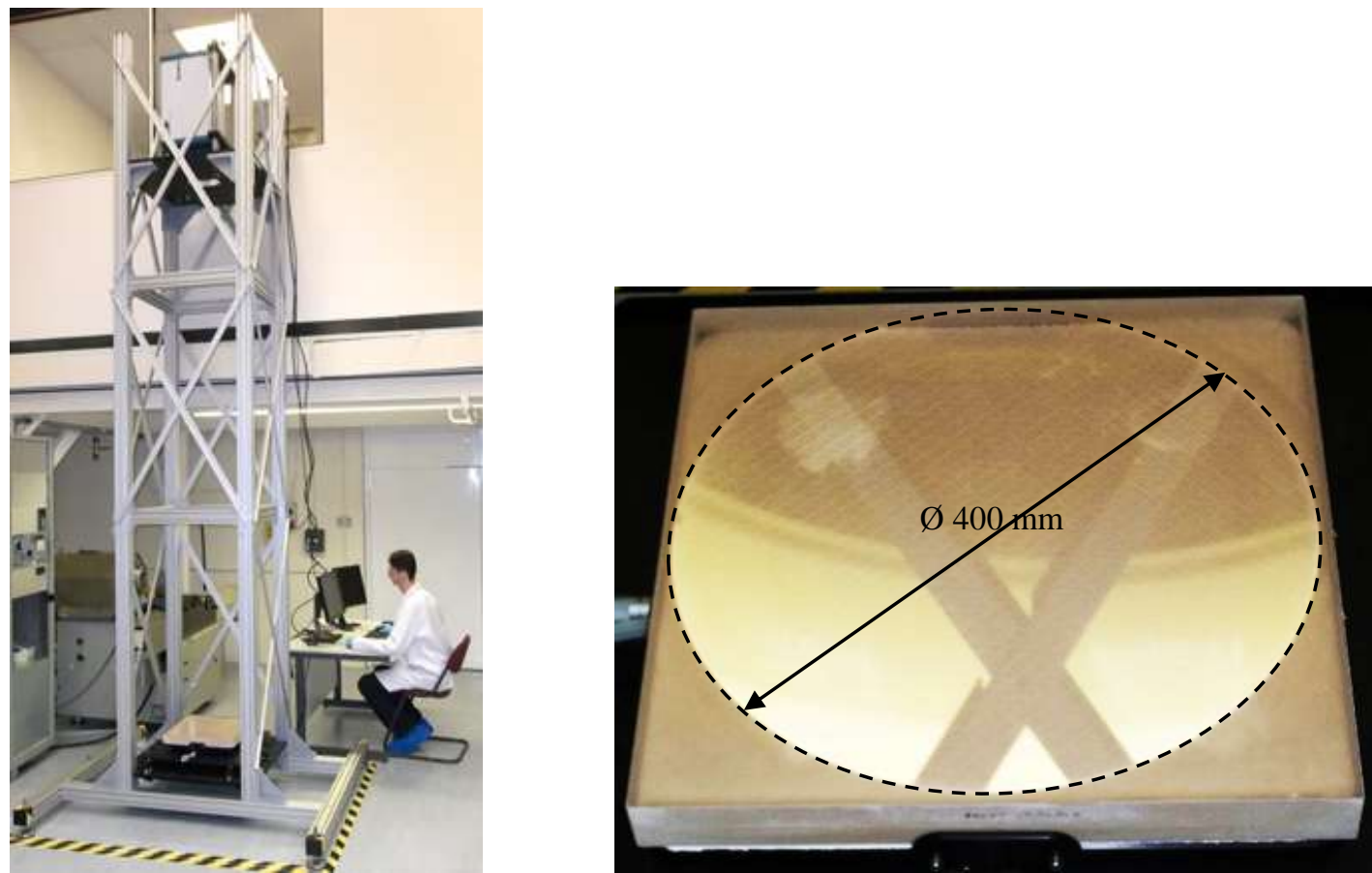

Figure 5. left: optical test-tower in the laboratory of Cranfield Precision Engineering; right: the ULE mirror on the stage of the optical test-tower.

\subsection{Figuring results}

The figure correction was performed following a two-cycle procedure that alternates rapid plasma figuring and "flash" polishing for roughness level restoration. In fact, a sequence that integrates a quick polishing process is considered the ultimate finishing procedure for a cost-effective optical fabrication chain. 
First, three plasma figuring iterations were carried out, which delivered a residual error of $\sim 43 \mathrm{~nm} \mathrm{rms}(280 \mathrm{~nm}$ PVr). The residual error map can be observed in Figure 6 (right). This procedure lasted 2.5 hours ( 51 minutes mean processing time), for an overall convergence of $89 \%{ }^{1}$.
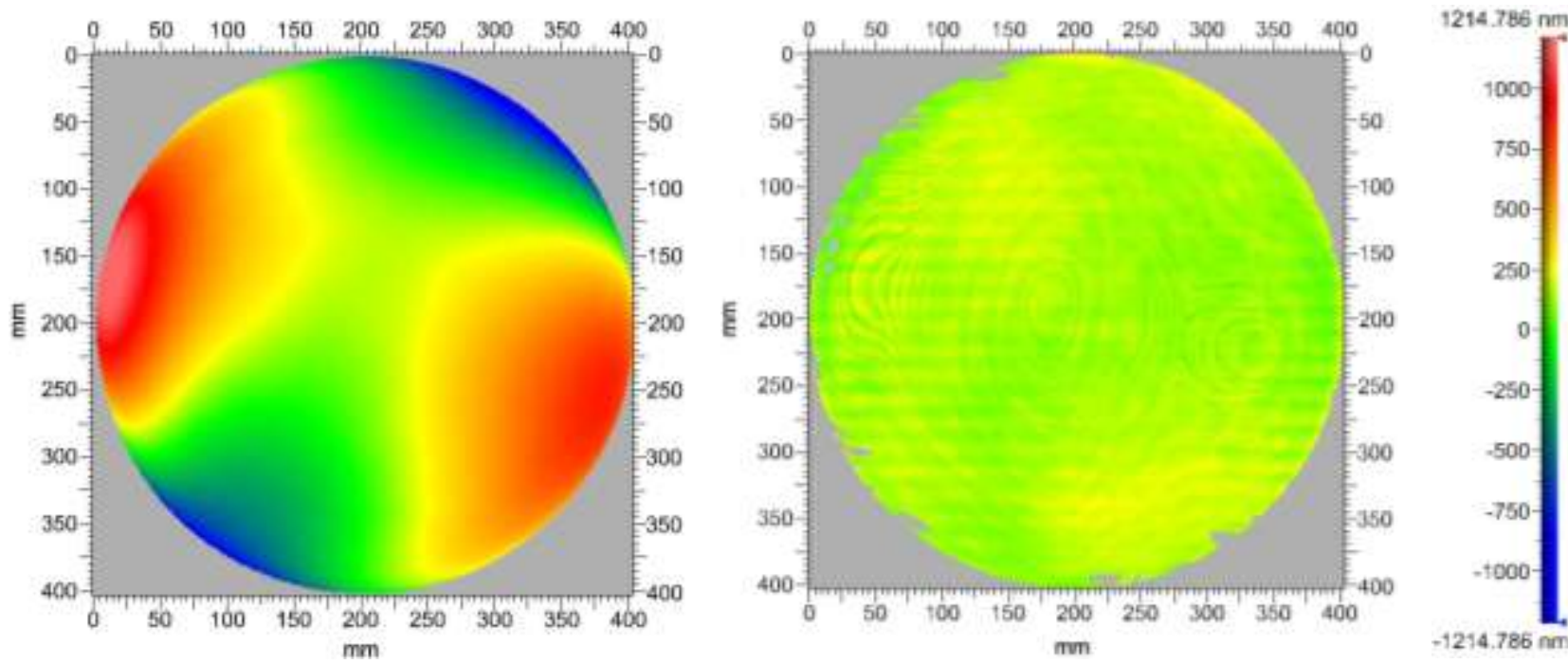

Figure 6. Initial (left) and final (right) figure error maps for the first figuring sequence.

At this stage, the second hand-polishing was carried out as the surface texture presented significant roughness increase, due to the large amount of material removed $(>2 \mu \mathrm{m})$. The post flash polishing figure error showed a loss of form accuracy up to $\sim 723 \mathrm{~nm}$ PVr (137 nm rms), as shown in Figure 7 (left). Therefore, two further plasma figuring iterations were necessitated to recover the form accuracy. These required a total processing time of 1.6 hours. Once again, the pitch sizes were alternated over the two consecutive iterations. The final residual figure error was $\sim 31 \mathrm{~nm} r m s ~(230 \mathrm{~nm} P V r)$, which corresponds to a $92 \%$ overall process convergence after $\sim 4$ hours when considering both figuring cycles. The final error map can be observed in Figure 7 (right). For both figuring procedures, the residual map showed no actual edge effect. In Table 1, some key data of the two figure correction cycles are summarized.
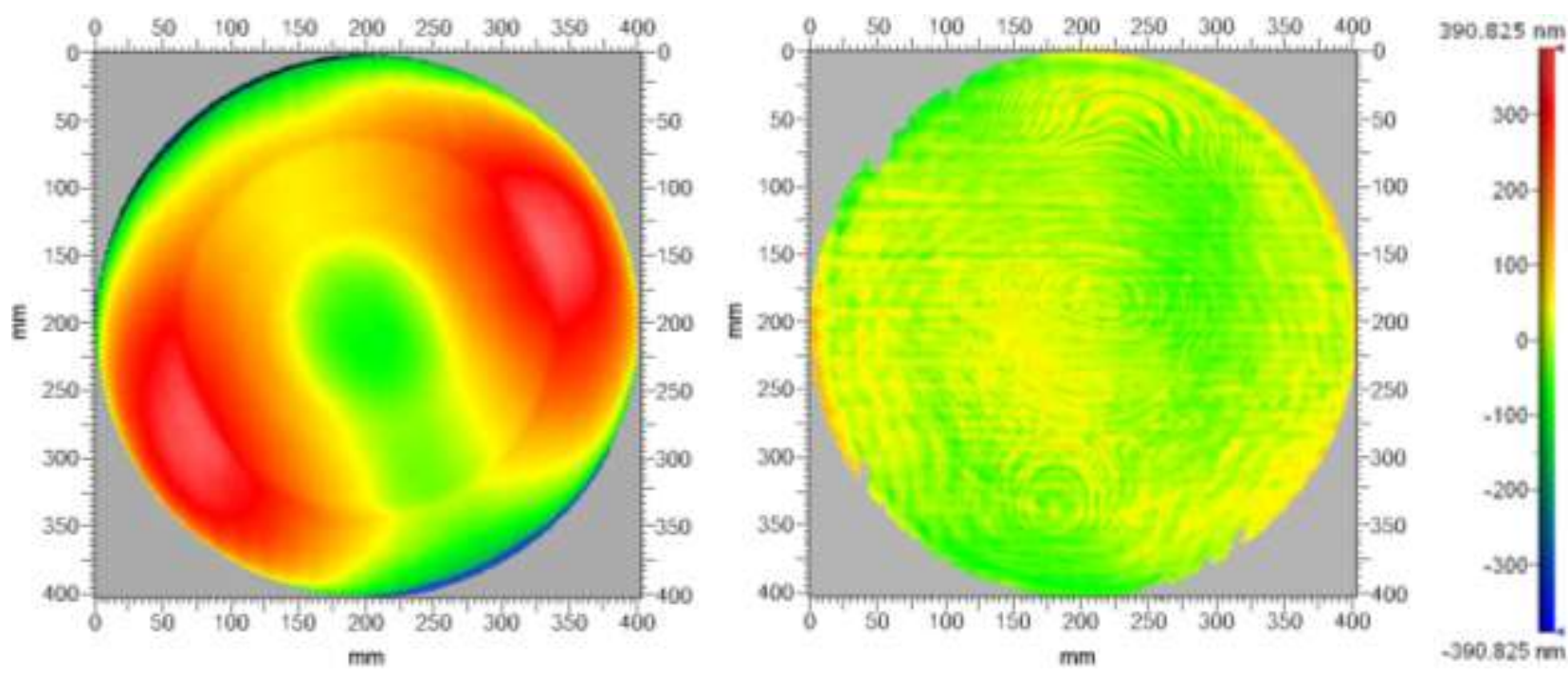

Figure 7. Initial (left) and final (right) figure error maps for the second figuring sequence.

${ }^{1}$ Defined on the basis of the rms convergence ratio [16]. 
From the presented results it is possible to estimate the processing times required for a meter-class surface characterized by $\sim 1 \mu \mathrm{m}$ PV figure error. If two iterations of $\sim 50$ minutes duration each are considered, the plasma figuring procedure would be completed in about ten hours. Additionally, the time for roughness level restoration should be accounted for. The introduction of a CNC controlled conformal sub-aperture "flash" polishing would deliver a high surface integrity, while preserving the form accuracy achieved by plasma figuring.

The residual error maps after the first and second figuring cycle present a patterned morphology. This is partially due to swirly features connected to material stress. However, the raster-scanning tracks of the plasma figuring can also be recognized (mid-spatial frequencies). These patterns have been investigated by means of Power Spectral Density (PSD) analysis. This is discussed in the next section.

Table 1. Summary of figuring processes.

\begin{tabular}{|c|c|c|}
\hline Evaluated aspect & $\begin{array}{c}\text { First figuring } \\
\text { procedure }\end{array}$ & $\begin{array}{l}\text { Second figuring } \\
\text { procedure }\end{array}$ \\
\hline Mean figuring time [ min/iteration] & 51 & 49 \\
\hline Total figuring time $[\mathrm{h}$ ] & 2.5 & 1.6 \\
\hline $\begin{array}{l}\text { Overall convergence [\% ] } \\
\text { (For the two cycles: } 92 \% \text { ) }\end{array}$ & 89 & 77 \\
\hline $\begin{array}{rr}\text { Figure error in rms }[\mathrm{nm}] & \text { Initial } \\
& \text { Final }\end{array}$ & $\begin{array}{c}373 \\
43\end{array}$ & $\begin{array}{c}137 \\
31\end{array}$ \\
\hline $\begin{array}{r}\text { Figure error in } \operatorname{PVr}[\mathrm{nm}] \quad \text { Initial } \\
\text { Final }\end{array}$ & $\begin{array}{c}2300 \\
280\end{array}$ & $\begin{array}{l}723 \\
230\end{array}$ \\
\hline
\end{tabular}

\section{POWER SPECTRAL DENSITY (PSD) ANALYSIS}

The RAP raster-scanning print-through was quantitatively assessed by calculating one-dimensional profile PSDs. These were taken across the known raster path direction, as illustrated by the arrow markers on the residual maps in Figure 8.
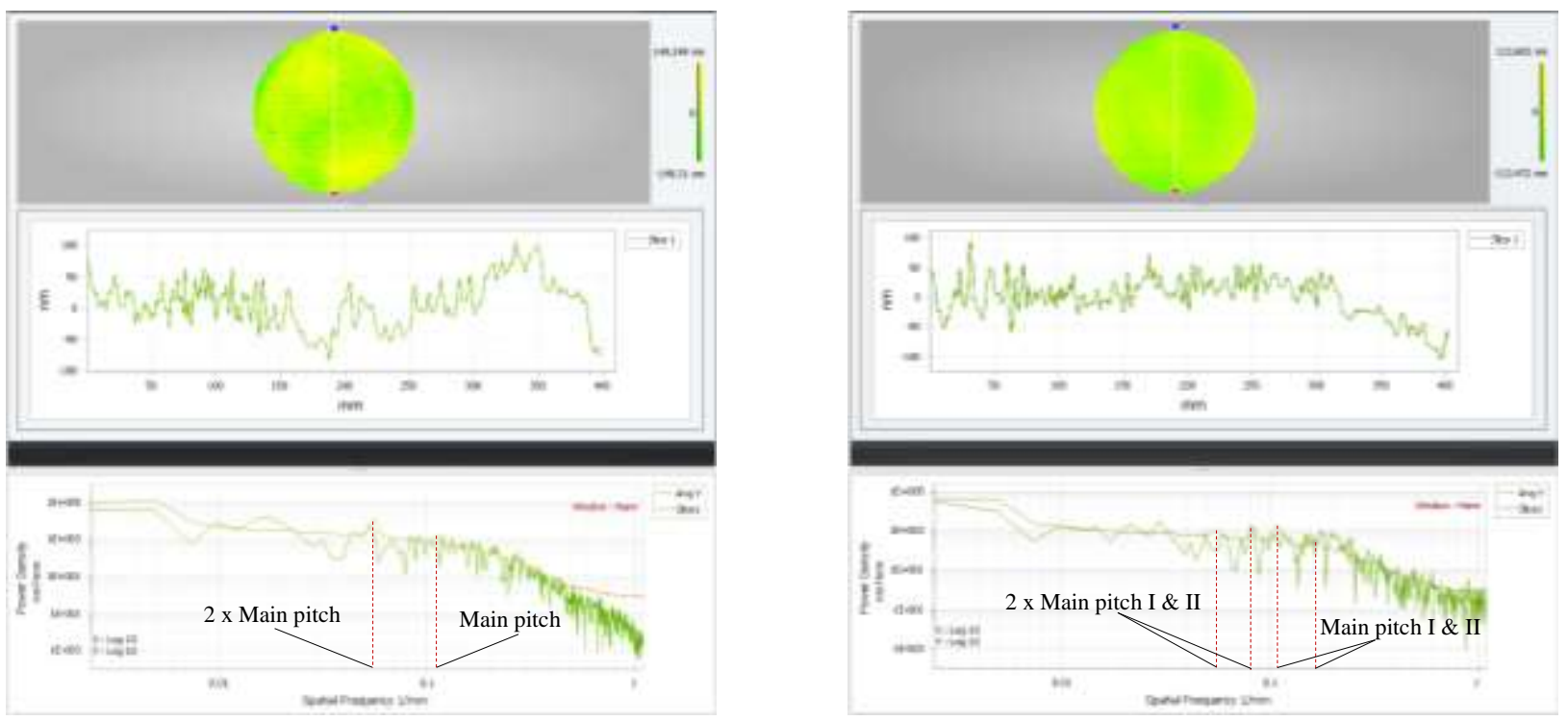

Figure 8. PSD analyses after first (left) and second (right) figuring cycle. 
In Figure 8, the PSD analysis of the residual figure error is shown for the final outcome of the two plasma processing cycles. Below the topography maps, exemplificative profile cross sections are displayed. They highlight the moderate final figure p-v: $150 \mathrm{~nm}$ along a surface cross section. Further, the figuring process output was analyzed using both single cross sections and average directional PSD. This has highlighted the presence of peaks which correlate the RAP main rastering pitch to the observed mid-spatial frequencies. As evidenced on these graphs, peaks matching the rastering main pitch are present. For the first figure correction cycle (Figure 8, left), they correspond to the size of staggering pitch and to twice this value. Conversely, the result of the second figuring procedure generates four peaks resulting from the overlapping of subsequent raster-scanning loops (Figure 8, right).

\section{CONCLUSIONS AND FUTURE WORK}

In this paper, the large scale figuring capability of the Reactive Atom Plasma process was demonstrated. A $\sim 400 \mathrm{~mm}$ diameter ULE mirror was figure corrected within a two cycle's procedure. During the first figuring sequence, the error was reduced by over $2 \mu \mathrm{m}$ and $\lambda / 15 \mathrm{rms}$ form accuracy was achieved in 2.5 hours (three iterations). At this stage, the mirror was flash polished. The surface figure was altered to $\sim 137 \mathrm{~nm} \mathrm{rms,} \mathrm{but} \mathrm{was} \mathrm{rapidly} \mathrm{corrected} \mathrm{by} \mathrm{applying} \mathrm{two}$ further plasma figuring iterations (1.6 hours). The final residual figure error resulted as $\lambda / 20 \mathrm{rms}$, for an overall process convergence of $92 \%$. From the processing times, it is possible to estimate the duration of the figure correction for a meter-class component: if $\sim 1 \mu \mathrm{m}$ PV figure error is assumed, a two-iteration procedure would deliver $\lambda / 20 \mathrm{rms}$ form accuracy in about 10 hours.

The surface topography presented a waviness patterns of the order of $\pm 30 \mathrm{~nm} \mathrm{p}-\mathrm{v}$, referable to the raster-scanning tracks. PSD analysis confirmed that they matched the main pitch sizes of the tool-path, as well as a twofold of the steps caused by overlapped staggering loops. The surface texture after plasma figuring showed an expected increase in roughness: this is typically due to chemical etching irregularities, enhanced in this case by the presence of subsurface damage. A "flash" polishing process is expected to significantly improve this aspect, as well as minimize ripples from the raster-patterns. Such a process is currently at the research phase at Cranfield University Precision Engineering. In combination with the plasma figuring procedure, this will constitute a fast and cost-effective finishing methodology that can be implemented into a rapid large optics fabrication chain.

\section{REFERENCES}

[1] Gilmozzi, R., "Science and technology drivers for future giant telescopes", Proc. SPIE 5489(1) 1-10 (2004).

[2] European Southern Observatory (ESO), "The European Extremely Large Telescope ("E-ELT") Project" 2 March 2012. <http://www.eso.org/sci/facilities/eelt/> (8 May 2012). http://www.eso.org/public/

[3] Spyromilio, J., Comeron, F., D'Odorico, S., Kissler-Patig, M., Gilmozzi, R., "Progress on the European Extremely Large Telescope", The Messenger 133, 2-8 (2008).

[4] TMT Project, "Thirty Metre Telescope", 8 May 2012. http://www.tmt.org/

[5] GMTO Corporation, "Giant Magellan Telescope", 8 May 2012. http://www.gmto.org/

[6] Shectman, S., Johns, M., "GMT overview," Proc. SPIE 7733(1), 77331Y (2010).

[7] Postman, M., Brown, T., Sembach, K., Giavalisco, M., Traub, W., Stapelfeldt, K., Calzetti, D., Oegerle, W., Rich, R. M., Stahl, H. P., Tumlinson, J., Mountain, M., Soummer, R., Hyde, T., "Science drivers and requirements for an Advanced Technology Large Aperture Space Telescope (ATLAST): Implications for technology development and synergies with other future facilities", Proc. SPIE 7731 77312K (2010)

[8] Shore, P., Cunningham, C., DeBra, D., Evans, C., Hough, J., Gilmozzi, R., Kunzmann, H., Morantz, P., Tonnellier, X., "Precision engineering for astronomy and gravity science," CIRP Annals - Manufacturing Technology 59, 694-716 (2010).

[9] Allen, L. N., "Progress in ion figuring large optics," Proc. SPIE 2428, 237-47 (1995).

[10] Messner, W., Hall, C., Dumas, P., Hallock, B., Tricard, M., O’Donohue, S., Miller, S., "Manufacturing meterscale aspheric optics," Proc. SPIE 671066671 (2007).

[11] Walker, D. D., Brooks, D., King, A., Freeman, R., Morton, R., McCavana, G., et al. "The 'Precessions' tooling for polishing and figuring flat, spherical and aspheric surfaces,” Optics Express 11(8), 958-64 (2003).

[12] Castelli, M., Jourdain, R., Morantz, P., Shore, P., "Rapid Optical Surface Figuring Using Reactive Atom Plasma," Precision Engineering 36(3), 467-476 (2012). 
[13] Evans, C. J. (2009), "PVr - a robust amplitude parameter for optical surface specification,” Optical Engineering 48(4) 043605.

[14] Comley, P., Morantz, P., Shore, P., Tonnellier X., "Grinding metre scale mirror segments for the E-ELT ground based telescope", CIRP Annals - Manufacturing Technology 60(1), 379-382 (2011).

[15] Subrahmanyan, P. K., Gardopée, G., "Reactive atom plasma (RAPTM) processing of mirrors for astronomy," Proc. SPIE 7018, 701809 (2008).

[16] Allen, L. N., Hannon, J. J., Wambach, R. W., "Final surface error correction of an off-axis aspheric petal by ion figuring," Proc. SPIE 1543, 190-200 (1992). 